\section{FORGOTTEN MEDICAL ROLE}

Sir, your editorial Periodontal diseases who cares? (BDJ 2011; 210: 565) is most timely. Not least, unlike some periodontists, in that you correctly use the term 'periodontal diseases'. Our many colleagues who are not periodontists have indeed the right to expect clear guidance from those particular specialists. Part of the problem, I suggest, is our failure as the dental profession to make patients aware that we are physicians as well as surgeons, and that while, like our other general medical colleagues we, too, have to diagnose as wide a range of disease as that affecting any other body organ or system, we have to spend most of our time with a patient in actual mechanical/surgical treatment. The result is that our medical role is forgotten, and few recognise the dentist as physician.

Perhaps the increasing evidence of the relevance of the chronic inflammatory periodontal diseases to a wide range of systemic diseases of high morbidity and mortality ${ }^{2}$ may concentrate the dental mind, and also open that mind to the wide range of non-CIPD diseases that can affect the periodontal diseases, ${ }^{3}$ and, additionally, renew the links between periodontology and oral medicine. As Victor Hugo said, nothing has the force of an idea whose time has come, and certainly periodontal medicine is here to stay. ${ }^{4}$ The primary concern of the periodontist, as of all of the dental team, must be the preservation of the natural dentition, for which titanium may not always be the best substitute, not least for an increasingly long-living population.

\section{H. N. Newman}

London

1. Newman $\mathrm{H}$ N. The dentist as physician. Br Dent J 1998; 185: 374

2. Newman H N. Focal infection. J Dent Res 1996; 75: 1912-1919.

3. Newman H N, Rees T D, Kinane D F. Diseases of the periodontium. Northwood, UK: Science Reviews Ltd, 1993.

4. Newman H N. Parodontalmedizin. Paradontologie 1994; 1: 61-64.

DOI: 10.1038/sj.bdj.2011.686

\section{READILY ACHIEVABLE}

Sir, in response to the editor's summary (BDJ 2011; 210: 418) of our recent manuscript, ${ }^{1}$ we suggest improved levels of instrument cleaning and sterility are readily achievable and that there is clear evidence of infection risks. The editor asks 'why are there concerns?' The manuscript references issues such as re-use of single use devices, inadequate segregation of clean and dirty instruments, failure to undertake periodic testing of equipment: all breaches of Health \& Safety regulations. These examples provide a robust evidencebase of poor practice potentially compromising patients and staff. ${ }^{2-5}$

Dental surgery is one of the few surgical specialities not performing surveillance for post-operative infections. Detecting transmission is reliant on practitioners submitting appropriate microbiological specimens. The absence of headlines, public health investigations or case reports should not be taken as grounds for complacency or absence of transmission. Infections, including MRSA, hepatitis B and vCJD, have an asymptomatic period ensuring that retrospective determination of adverse events is exceptionally difficult. This is reflected in the paucity of data relating instrument decontamination protocols to clinical outcomes. In dentistry there are additional confounding variables: co-morbidities; type and duration of intervention; difficulty in distinguishing endogenous and exogenous infection and the number of adverse events required for reliable assessment. Basing clinical decisions on a lack of transmission evidence when it has not been actively sought is unwise.

The editor raises pertinent questions regarding clean and unclean. The International Standard on automated washer disinfectors (AWDs) ${ }^{6}$ sets out definitions and processes comprising specification, installation, operation and performance qualification. Effective performance of AWDs relies on these processes and operator vigilance. While acceptable levels of cleanliness of surgical instruments is still debated, a sensible starting point is visibly clean instruments when inspected under illuminated magnification - a standard that patients would clearly grasp. That this standard was met in the majority of practices assessed confirms its achievability and that small, practical changes elsewhere would give universal compliance.
Lastly, the editor describes drives to improve reusable instrument decontamination standards as a 'futile pursuit of unrealistic sterility'. The paper focussed on evaluating dental instrument cleaning efficacy. This is linked to instrument sterility in two ways: dirty instruments take longer to sterilise reducing safety margins and provide a matrix for microbial re-colonisation if sterile packaging is compromised. In relation to vCJD, the prion agent is less susceptible to steam inactivation, so efficient cleaning combined with steam sterilisation is essential to minimise transmission risks. While the precise level of acceptable residual protein on cleaned instruments is debatable, variability between practices is not acceptable and clearly addressable. The concept of 'unrealistic sterility' is not a futile objective and the technical definition (eg HTM 01-05, IS0-17665-1:2006 and/or BS-EN-556-1:2001) of surgical instrument sterility is routinely achieved in many aspects of surgery, including general dental practice. Technical support to GDPs is essential as emphasised in our concluding sentence.

We applaud the editor for asking whether there is achievable room for improvement and evidence that it will enhance patient and staff protection. We suggest the case made in the manuscript and cited documents is clear and affirmative.

J. M. Sutton, J. T. Walker, N. D. H. Raven, P. Marsh, A. Smith, D. Perrett By email

1 Vassey M, Budge C, Poolman T et al. A quantitative assessment of residual protein levels on dental instruments reprocessed by manual, ultrasonic and automated cleaning methods. Br Dent J 2011; 210: E14.

2. Redd J T, Baumbach J, Kohn W, Nainan O, Khristov M, Williams I. Patient-to-patient transmission of hepatitis B virus associated with oral surgery. $J$ Infect Dis 2007; 195: 1311-1344.

3. Martin M V, Hardy P. Two cases of oral infection by Methicillin resistant Staphylococcus aureus. Br Dent J 1991; 170: 63-64.

4. Kurita H, Kurashina K, Honda T. Nosocomial transmission of methicillin resistant Staphylococcus aureus via the surfaces of the dental operatory. Br Dent J 2006; 201: 297-300

5. Rokadiya S, Malden N J. An implant periapical lesion leading to acute osteomyelitis with isolation of Staphylococcus aureus. Br Dent J 2008; 205: 489-491.

6. BS EN ISO 15883-1:2006 Washer-disinfectors - Part 1: General requirements, terms and definitions and tests. Available at: http://www.iso.org/ iso/catalogue_detail.htm?csnumber $=41076$

DOI: $10.1038 /$ sj.bdj.2011.687 